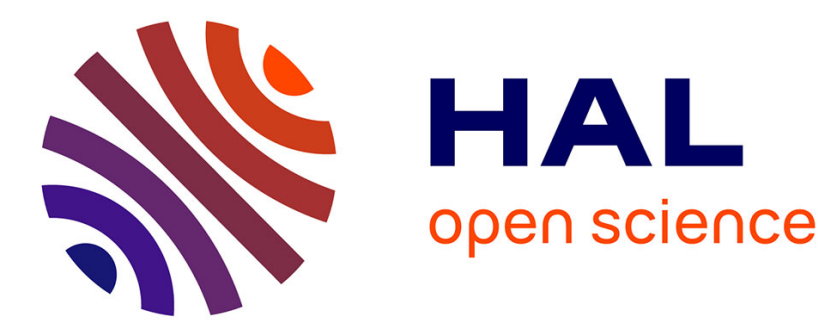

\title{
The potentially hazardous Asteroid (214869) 2007 PA8: An unweathered $L$ chondrite analog surface
}

\author{
S Fornasier, Irina N. Belskaya, D Perna
}

\section{To cite this version:}

S Fornasier, Irina N. Belskaya, D Perna. The potentially hazardous Asteroid (214869) 2007 PA8: An unweathered L chondrite analog surface. Icarus, 2015, 250, pp.280 - 286. 10.1016/j.icarus.2014.12.015 . hal-01103405

\section{HAL Id: hal-01103405 \\ https://hal.sorbonne-universite.fr/hal-01103405}

Submitted on 14 Jan 2015

HAL is a multi-disciplinary open access archive for the deposit and dissemination of scientific research documents, whether they are published or not. The documents may come from teaching and research institutions in France or abroad, or from public or private research centers.
L'archive ouverte pluridisciplinaire HAL, est destinée au dépôt et à la diffusion de documents scientifiques de niveau recherche, publiés ou non, émanant des établissements d'enseignement et de recherche français ou étrangers, des laboratoires publics ou privés. 


\title{
The potentially hazardous asteroid (214869) 2007 PA8: an unweathered $\mathrm{L}$ chondrite analogue surface
}

\author{
S. Fornasier ${ }^{1,2}$, I.N. Belskaya ${ }^{1,3}$, D. Perna ${ }^{1}$
}

${ }^{1}$ LESIA, Observatoire de Paris, CNRS, UPMC Univ Paris 06, Univ. Paris Diderot, 5 Place J. Janssen, 92195 Meudon Pricipal Cedex, France

${ }^{2}$ Univ. Paris Diderot, Sorbonne Paris Cité, 4 rue Elsa Morante, 75205 Paris Cedex 13

${ }^{3}$ Astronomical Observatory of Kharkiv National University, 35 Sumska str., 61022

Kharkiv, Ukraine Submitted to Icarus: September 2014

e-mail: sonia.fornasier@obspm.fr; fax: +33145077144; phone: +33145077746

Manuscript pages: 25; Figures: 5; Tables: 4

\section{Running head: The PHA 2007 PA8}

Send correspondence to:

Sonia Fornasier

LESIA-Observatoire de Paris

Batiment 17

5, Place Jules Janssen

92195 Meudon Cedex

France

e-mail: sonia.fornasier@obspm.fr

fax: +33145077144

phone: +33145077746 


\title{
The potentially hazardous asteroid (214869) 2007 PA8: an unweathered $\mathrm{L}$ chondrite analogue surface
}

\author{
S. Fornasier ${ }^{1,2}$, I.N. Belskaya ${ }^{1,3}$, D. Perna ${ }^{1}$
}

\begin{abstract}
In this paper we present the results on the polarimetric and spectroscopic observations of the Potentially Hazardous Asteroid (214869) 2007 PA8 obtained during its favorable apparition in October-November 2012, when it approached the Earth at the minimal distance of $0.043 \mathrm{AU}$. Polarimetry was carried out at the NOT in the B, V, R, and I bands covering both low $\left(12-23^{\circ}\right)$ and large phase angles $\left(88-99^{\circ}\right)$. Spectroscopy in the visible and near infrared range was obtained at the TNG telescope.

The spectrum of 2007 PA8 shows silicates absorption features and a behavior consistent with a Q-type classification. The olivine and pyroxene BI band is centered at $0.9578 \pm 0.0042 \mu \mathrm{m}$, with a band depth of $16.5 \%$, the BII band is centered at $1.95 \pm 0.01 \mu \mathrm{m}$, and it has a band depth of about 3.9\%. The 2007 PA8 spectral parameters are consistent with those of L chondrites. Also the spectral comparison with meteorites gives the L-type chondrites, and L6 in particular, as best match.

The NEA (214869) 2007 PA8 is the forth moderate albedo asteroid and the first Q-type asteroid for which the value of the polarization maximum is determined. The inversion angle of the polarization curve in the $\mathrm{V}$ filter is $19.0 \pm 1.1^{\circ}$, the corresponding slope parameter $(h)$ is of $0.078 \pm 0.010 \% /^{\circ}$, the maximum value of polarization is $5.99 \pm 0.16 \%$, and the extreme value of negative polarization is estimated to be lower than $-0.52 \%$. Using the polarimetric slope we derive a geometric albedo of $\mathbf{0 . 2 9} \pm \mathbf{0 . 0 8}$ in the $\mathrm{V}$ band, that gives an estimated diameter of $\mathbf{1 . 4} \pm \mathbf{0 . 2}$ $\mathbf{k m}$, assuming an absolute $\mathrm{H}_{v}$ magnitude of $16.2 \mathrm{mag}$. We find a strong dependence of the polarization in the $\mathrm{B}, \mathrm{V}, \mathrm{R}$, and I bands with wavelength, and the polarimetric albedo in the four bands is strongly correlated with the asteroid's spectrum. The 2007 PA8 polarimetric properties resemble those of other 2 NEAs, 1566 Icarus and 25143 Itokawa, which are both S(IV)/Q type. Our spectral and polarimetric analysis indicate that 2007 PA8 has a young and fresh surface almost unweathered, similar to L-type chondrites. These results, together with dynamical simulations made by Nedelcu et al. (2014) and Nesvorny et al. (2009), indicate that 2007 PA8 may be a member of the Gefion family recently ejected from the 5:2 resonance and a potential source of L chondrites.
\end{abstract}

Keywords: Near-Earth objects, Asteroids, Surfaces, Spectroscopy, Polarimetry, Meteorites, Asteroids, composition 


\section{Introduction}

Potentially hazardous asteroids (PHA) are near earth asteroids (NEA) whose minimum orbit intersection distance with the Earth is $0.05 \mathrm{AU}$ or less and whose absolute magnitude $\mathrm{H}_{v}$ is 22.0 or brighter. The knowledge of their physical properties (size, albedo, density, surface mineralogical composition) is very important to define a successful mitigation strategy, since the technology needed to deflect or disrupt a potential impactor depends on its physical properties (Perna et al. 2013). The entire population of NEAs is supposed to contain about 1000 objects with diameter larger than $1 \mathrm{~km}$ and more than twenty of thousands greater than $100 \mathrm{~m}$ (Mainzer et al. 2011). NEAs are responsible for most meteorite falls and for the occurrence of occasional major catastrophic impact events. Our knowledge of the structure and composition of NEAs is still rather limited, since only $\sim 5 \%$ of the known NEAs have spectral types determined from observations. The knowledge of the chemical and mineralogical composition of NEAs surfaces gives insight on the materials which formed the protoplanetary nebula at different solar distances, and on the processes that governed the formation and the evolution of our planetary system.

Asteroid (214869)2007 PA8 is a PHA discovered on 09 August 2007 by the LINEAR sky survey. It is an Apollo Earth-crossing asteroid with a minimum orbit intersection distance (MOID) of 3.59 millions of $\mathrm{km}$ (0.024 AU). It is also a target of relatively easy access for a space mission, having a minimum $\Delta \mathrm{V}$ of $6.8 \mathrm{~km} / \mathrm{s}$ (computed following the method by Shoemaker and Helin, 1978). 2007 PA8 has a Tisserand invariant $\mathrm{T}_{J}=2.95$, and it resembles that of a Jupiter-family comet. Its physical and orbital parameters are summarized in Table 1. Hicks et al. (2012) got Bessel B, V, R, and I observations of this PHA finding a long rotational period of 
95.1 $\pm 3.4 \mathrm{hr}$ and visible colors compatible with a $\mathrm{C}$ or Xc-type spectral classification following the Tholen or the Bus-Demeo taxonomy (Tholen \& Barucci, 1989; Demeo et al. 2009). Assuming a geometric albedo of 0.05, typical for C-type asteroids, they compute an effective diameter of $\sim 3 \mathrm{~km}$. Extensive radar observing campaign were performed at the Goldstone radar on October-November 2012 . Radar images reveal an irregular and asymmetric shape with rough dimensions of $1.9 \mathrm{~km} \times 1.4 \mathrm{~km} \times 1.3 \mathrm{~km}$, the presence of possible craters and boulders, and a very slow rotation $(102 \pm 2 \mathrm{hr}$ ) in retrograde spin state (Brozovic et al., 2013).

We present in this paper the results of the 2007 PA8's polarimetric and spectroscopic observations obtained during its favorable apparition in October-November 2012, when it approached the Earth at the minimal distance of $0.043 \mathrm{AU}$ (the next close approach of this asteroid to the Earth will be in 2036). Our data allow to constrain the surface composition of this PHA, to obtain the first measurement of its albedo, and thus to determine its size.

\section{Observations and data reduction}

\subsection{Spectroscopy}

Spectroscopic observations in the visible and near infrared range of 2007 PA8 were made at the $3.56 \mathrm{~m}$ Italian Telescopio Nazionale Galileo (TNG) of the European Northern Observatory (ENO) in la Palma, Spain, on 12-13 October 2012. For visible spectroscopy we used the Dolores (Device Optimized for the LOw RESolution) instrument equipped with the low resolution red (LRR) and blue (LRB) grisms. The LRR grism covers the $0.52-0.95 \mu \mathrm{m}$ range with a spectral dispersion of $2.9 \AA / \mathrm{px}$, while the LRB grism covers the $0.38-0.80 \mu \mathrm{m}$ range with a spectral dispersion of $2.8 \AA / \mathrm{px}$. The Dolores detector is a Loral thinned and 
back-illuminated CCD with $2048 \times 2048$ pixels, with a pixel size of $15 \mu \mathrm{m}$ and a pixel scale of $0.275 \mathrm{arcsec} / \mathrm{px}$. The 'red' and 'blue' spectra in the visible range were separately reduced and finally combined together to obtain spectral coverage from 0.38 to $0.95 \mu \mathrm{m}$.

For the infrared spectroscopic investigation we used the near infrared camera and spectrometer (NICS) equipped with an Amici prism disperser. This equipment covers the $0.85-2.40 \mu \mathrm{m}$ range during a single exposure with a spectral resolution of about 35 (Baffa et al. 2001). The detector is a $1024 \times 1024$ pixel Rockwell HgCdTe Hawaii array. The spectra were acquired nodding the object along the spatial direction of the slit, in order to obtain alternating pairs (named A and B) of near-simultaneous images for the background removal. For both the visible and near infrared observations we utilized a 2 arcsec wide slit, oriented along the parallactic angle to minimize the effect of atmospheric differential refraction.

Visible and near-infrared spectra were reduced using ordinary procedures of data reduction with the software packages Midas as described in Fornasier et al. $(2004,2010)$. For the visible spectra, the procedure includes the subtraction of the bias from the raw data, flat-field correction, cosmic ray removal, sky subtraction, collapsing the two-dimensional spectra to one dimension, wavelength calibration, and atmospheric extinction correction. The spectra were normalized at $6000 \AA$. For observations in the infrared range, spectra were first corrected for flat fielding, then bias and sky subtraction was performed by producing A-B and B-A frames. The positive spectrum of the B-A frame was shifted and added to the positive spectrum of the A-B frame. The spectrum was then extracted and wavelength calibrated. For both visible and near infrared observations the reflectivity of 2007 PA8 was obtained by dividing its spectrum by that of a solar analog star, observed 
close in time and in airmass to the object. The infrared and visible spectral ranges were combined by overlapping the spectra, merging the two wavelength regions at the common wavelengths and utilizing the zone of good atmospheric transmission to find the normalization factor between the two spectral parts.

Details on the observing conditions are reported in Table 2.

\subsection{Polarimetry}

Polarimetric observations were carried out at the 2.5 Nordic Optical Telescope (NOT) in La Palma, Spain, during 4 runs between 13 October and 14 November 2012. The observations were done in the Bessel B, V, R and I filters using the Wedged Double Wollaston prism (Oliva, 1997) mounted on the ALFOSC instrument. The detector is a back illuminated CCD of $2048 \times 2048$ pixel (pixel size of $13.5 \mu \mathrm{m})$, providing a total field of view of $6.5 \times 6.5 \mathrm{arcmin}$, with a pixel scale of $0.19 \mathrm{arcsec} / \mathrm{px}$. This polarimeter, previously mounted at the $1.82 \mathrm{~m}$ Asiago telescope, splits the incoming light into four polarized beams at $0^{\circ}, 45^{\circ}, 90^{\circ}$, and $135^{\circ}$, separated by 20 arcsec (Pernechele et al. 2003). These four beams are sufficient to determine simultaneously the linear polarization parameters I, Q, and U with a single exposure. This instrument was tested successfully for several polarimetric observations of minor bodies as shown in Fornasier et al. (2006), and Belskaya et al. (2009).

During each run, the observational procedure includes bias and flat field acquisitions, together with the observation of polarized and unpolarized standard stars to calibrate the instrumental polarization. The flat field images were acquired in two different sets corresponding to the adapter position of $0^{\circ}$ and $90^{\circ}$, to average the polarization induced by the reflections on the dome screen of the flat field lamp. These 2 sets were first bias subtracted, then added to produce a master flat. 
The asteroid and standard stars images were corrected for bias and master flat, then aperture photometry was applied. The flux for each channel was integrated over a radius corresponding to 3-4 times the average seeing, and the sky was subtracted using a 3-5 pixel wide annulus around the asteroid. Naming $\mathrm{I}_{0}, \mathrm{I}_{90}, \mathrm{I}_{45}$, and $\mathrm{I}_{135}$, the flux corresponding to the channels at $0,90,45$, and $135^{\circ}$ of polarization, the Stokes parameters have been derived as follows:

$$
\begin{gathered}
Q=\frac{I_{90}-I_{0}}{I_{90}+I_{0}} \\
U=\frac{I_{135}-I_{45}}{I_{135}+I_{45}}
\end{gathered}
$$

The degree of polarization $P$ and the position angle $\theta$ of the polarization plane in the instrumental reference system are expressed via the parameters $Q$ and $U$ with the well-known formulae

$$
\begin{aligned}
& P=\sqrt{U^{2}+Q^{2}} \quad \sigma_{P}=\frac{|U * d U+Q * d Q|}{P} \\
& \theta=\frac{1}{2} \arctan \frac{U}{Q} \quad \sigma_{\theta}=\frac{28.65 * \sigma_{P}}{P}
\end{aligned}
$$

where dU and dQ are the errors on the Stokes parameters (Shakhovskoy \& Efimov, 1972).

The position angle of the polarization plane relative to the plane perpendicular to the scattering plane, $\theta_{r}$, is expressed in terms of the position angle $\phi$ as

$$
\theta_{r}=\theta-\left(\phi \pm 90^{\circ}\right)
$$

where the sign inside the bracket is chosen to assure the condition $0^{\circ} \leq\left(\phi \pm 90^{\circ}\right) \leq$ 
$180^{\circ}$. The polarization quantity $P_{r}$ is derived from

$$
P_{r}=P * \cos \left(2 \theta_{r}\right)
$$

The observing conditions and the final results on the degree of polarization $P$, on the position angle $\theta$ of the polarization plane, and on the polarimetric quantities $P_{r}$ and $\theta_{r}$ are reported in Table 3.

\section{Results}

\subsection{Taxonomy and composition}

The two visible spectra acquired at the TNG telescope are shown in Fig. 1, together with the albedo values, normalized in the $\mathrm{V}$ band, derived from $\mathrm{B}, \mathrm{V}, \mathrm{R}$ and I polarimetry. The two spectra are taken 72 minutes apart and they are very similar, showing that the surface sampled during these observations is homogeneous.

The combined visible and infrared spectra of 2007 PA8 (Fig. 2) clearly show the presence of silicate bands in the $0.9-1 \mu \mathrm{m}$ and $2 \mu \mathrm{m}$ region, and an overall behavior consistent with a Q-type asteroid classification, following the Bus \& DeMeo classification scheme (DeMeo et al., 2009). Within the S-complex, Q-type asteroids match ordinary chondrite spectra, and therefore have surfaces that appear young, fresh, and so unweathered, implying they have been recently exposed (DeMeo et al. 2014).

We analyzed the spectrum following the procedure described by Gaffey et al. (1993). We find a BI band center of $0.9578 \pm 0.0042 \mu \mathrm{m}$, with a band depth of $16.5 \%$. This band is due to the presence of both olivine and pyroxene. Beyond $1.8 \mu \mathrm{m}$ the spectrum is quite noisy, so we apply a order 2 polynomial fit to the data 
in the 1.7-2.3 $\mu \mathrm{m}$ region to determine the BII band spectral parameters. The BII band is centered at $1.958 \pm 0.015 \mu \mathrm{m}$ and has a band depth of about 3.9\%. Nevertheless the upper edge of the 2 micron band is not well defined, so the band II area is probably underestimated.

The derived $2 \mu \mathrm{m} / 1 \mu \mathrm{m}$ band area ratio is $0.4 \pm 0.1$. Following the Dunn et al. (2010, 2013) formulas that relate mineral abundances with band BI center and with $2 \mu \mathrm{m} / 1 \mu \mathrm{m}$ band area ratio, we find a fayalite abundance (Fa) of $23.1 \pm 0.8$ mol\% in olivine, a ferrosilite abundance $(\mathrm{Fs})$ of $19.7 \pm 0.6 \mathrm{~mol} \%$ in pyroxene, and an olivine on olivine plus pyroxene abundance of $0.63 \pm 0.02$.

On the basis of these values 2007 PA8 is placed in the space of ordinary chondrites, and, in particular, comparing them with those of L, LL, and $\mathrm{H}$ chondrites (Fig. 2 in Dunn et al., 2013), it appears that the NEA has spectral parameters consistent with the L chondrites.

\subsection{Meteorite analogs}

To constrain the possible mineralogy of 2007 PA8 we conducted a search for meteorite spectral analogs using the RELAB spectrum library (Pieters and Hiroi 2004), and following the same procedure explained in Fornasier et al. (2010, 2011). For each spectrum in the library, a filter was applied to find relevant wavelengths ( 0.4 to $2.45 \mu \mathrm{m})$. Then, a second filter was applied to reject spectra with irrelevant albedo values (i.e. brightness at $0.55 \mu \mathrm{m}<0.15$ and $>0.4$ ). A Chisquared value was calculated relative to the normalized input asteroid spectrum. We used the asteroid wavelength sampling to resample the laboratory spectra by linear interpolation. This allowed a least-squares calculation with the number of points equal to the wavelength sampling of the asteroid spectrum. The RELAB data files were sorted according to the corresponding Chi-squared values, and 
finally we visually examined them for dynamic weighting of spectral features.

The two best spectral matches are represented in Fig. 2: both meteorites are Ltype chondrites, in agreement with the spectral analysis of 2007 PA8 that already pointed versus a L-type spectral analogy for this PHA. The best match is given by the Vouille L6 ordinary chondrite, which has an albedo of 0.24 , and the second best fit by another L6 chondrite, Denver, which has an albedo of 0.34 . Both samples have grain size $<150$ micron, and are ordinary chondrite with almost all metal removed (Jarosewich, 1990).

\subsection{Albedo and polarimetric properties}

The NEA (214869) 2007 PA8 was observed in BVRI bands during five nights in October-November 2012 (Table 3), covering both low $\left(12-23^{\circ}\right)$ and large phase angles $\left(88-99^{\circ}\right)$. The measured polarization phase curve is shown in Fig. 3. We find a strong dependence of the polarization with wavelength. These data allow us to estimate the polarization maxima and minima, and the polarimetric slopes for B, V, R, and I bands. The polarimetric slope was calculated by a linear fit to the observations at small phase angles. Since there are only three phase angles covered, the uncertainties of slope's values are rather large. The whole range of phase angles were fitted by the Lumme and Muinonen function (Lumme \& Muinonen, 1993):

$$
P(\alpha)=b(\sin \alpha)^{c_{1}}(\cos (0.5 \alpha))^{c_{2}} \sin \left(\alpha-\alpha_{0}\right)
$$

where we fixed two parameters: the parameter $c_{2}=0.35$ and the inversion angle $\alpha_{0}$ which was estimated from the linear fit. In Fig. 3 we show the fit for the $\mathrm{B}$ and $\mathrm{R}$ bands (dashed lines) just to demonstrate the predicted behavior at large phase angles. We assumed that our measurements at the phase angle of 
$99.2^{\circ}$ are close to the polarization maximum. In Table 4 we report our estimations of $\mathrm{P}_{\min }$, inversion angle, polarimetric slope $(\mathrm{h}), \mathrm{P}_{\max }$, and their uncertainties for each band.

We use the measured polarimetric slope to calculate the asteroid's albedo $\left(p_{v}\right)$. Albedo from the polarimetric slope was determined using well-known empirical relationship h-albedo:

$$
\log p_{v}=C_{1} \log h+C_{2}
$$

The coefficients $\mathrm{C}_{1}$ and $\mathrm{C}_{2}$ are slightly different according to the different albedo datasets used to calibrate this relationship. We utilize $\mathrm{C}_{1}=\mathbf{- 1 . 1 1 8} \pm \mathbf{0 . 0 7 1}$ and $\mathrm{C}_{2}=-1.779 \pm 0.062$ given by Cellino et al. (1999), and we derive a geometric albedo of $0.29 \pm 0.08$ in the $\mathrm{V}$ band. The obtained albedo values in the BVRI bands with their errors are given in Table 4.

We have also analyzed how the calibration of the relationship h-albedo influence the values of albedos. We found that the available calibrations based on asteroid data (Lupishko and Mohamed, 1996; Cellino et al. 1999, 2012; Masiero et al. 2012) gave consistent results within the uncertainties associated to the polarimetric slope h. The calibration based on meteorite data (Zellner \& Gradie 1976) gave smaller values of albedos $(0.21 \pm 0.03$ in the $\mathrm{V}$ band $)$, but it must be noted that the meteorites' albedos were measured at the phase angles of $5^{\circ}$ (see e.g. Geake \& Dollfus, 1986) and not at zero phase angle, as normally defined for asteroids' albedo. Considering that moderate-albedo asteroids show an increase in brightness at zero phase angle due to the opposition effect of about 1.4 times their brightness observed at phase $5{ }^{\circ}$ (see Belskaya \& Shevchenko, 2000), the geometric albedo inferred from the polarimetric albedo derived from the Zellner and Gradie (1976) relationship is about 0.29 , consistent with 
our adopted value.

The determined value of the asteroid's albedo perfectly matches the mean albedo value for Q-type asteroids (Thomas et al., 2011). The obtained values of albedos in the BVRI bands well reproduce the spectral behavior measured in the visible wavelengths (Fig. 1).

We estimated the asteroid's absolute magnitude analyzing the photometric observations made by Hicks et al. (2012) in the phase angle range 6.4-37.6 ${ }^{o}$. We first did a linear fit of these data, then we corrected the linear magnitude for the opposition effect, estimated to be $\sim \mathbf{0 . 3 5} \pm \mathbf{0 . 0 4}$ mag for moderate albedo asteroids (Belskaya $\&$ Shevchenko, 2000). The resulting absolute magnitude $H_{v}$ is $16.2 \pm 0.1 \mathrm{mag}$, where the uncertainty takes into account the errors of the linear fit and of the opposition effect. Using this $\mathbf{H}_{v}$ value and the V-band albedo previously determined, the estimated 2007 PA8 diameter is $1.4 \pm 0.2 \mathrm{~km}$.

At present the polarization maximum was measured for very few NEAs (e.g. De Luise et al. 2007, Belskaya et al. 2009 and references therein). The NEA (214869) 2007 PA8 is the forth moderate albedo asteroid and the first Q-type for which the value of polarization maximum has been determined. In Figure 4 we plot all the available polarimetric data for NEAs with moderate albedo values. We used only the $\mathrm{V}$ band data to avoid differences due to wavelength dependence of polarization. The value of $\mathrm{P}_{\max }$ for $2007 \mathrm{PA} 8$ is the lowest one compared to other silicate rich asteroids at the same phase angle of $\sim 100 \mathrm{deg}$.

Considering that the $\mathrm{P}_{\max }$ value strongly anticorrelates with albedo, this means that the surface of 2007 PA8 is brighter compared to those of 2000 PN9, Toutatis and Toro. There are systematic differences between polarization measured for 
these asteroids and that of our target, assuming that the surface of 2007 PA8 is brighter. On the other hand, the polarization degrees measured for 2007 PA8 are close to those measured for Icarus and Itokawa, indicating that these bodies have similar surface properties. These asteroids also show similar polarization wavelength dependence (Fig. 5). Moreover the geometric albedo of Itokawa and Icarus, 0.29 (Bernardi et al. 2009) and 0.33 (Harris, 1998), respectively, are very close to the one here determined for 2007 PA8.

To estimate the albedo from the $\mathrm{P}_{\max }$ value we used the empirical relationship:

$$
\log P_{\max }=C_{3} \log p_{v}+C_{4}
$$

based on measurements of lunar fines and terrestrial samples of different grain sizes (Geake \& Dollfus 1986). The best match of the 2007 PA8 V-band albedos defined from polarimetric slopes and from $\mathrm{P}_{\max }$ occurred for $\mathrm{C}_{3}=-0.73$ and $\mathrm{C}_{4}=-$ 0.13 . These coefficients are intermediate between those found for lunar fines with grain sizes $<50 \mu \mathrm{m}$, and for terrestrial samples with grain sizes $\sim 200-340$ microns (Geake \& Dollfus, 1986).

\section{Summary and Discussion}

From our studies the PHA 2007 PA8 is a Q-type asteroid with a fresh unweathered surface, a spectrum similar to the $\mathrm{L}$ chondrites, and with polarimetric properties analogues to the NEAs Itokawa and Icarus, both classified as S(IV)/Qtype asteroids (DeMeo et al. 2014, Binzel et al. 2001).

Nedelcu et al. (2014) got a spectrum of 2007 PA8 in the NIR region (0.8-2.4 $\mu \mathrm{m})$ from the IRTF telescope one month before our observations. Their spectrum is different compared to the one here presented, with a deeper 2 micron band, and 
with band I and II center positions at $0.92 \pm 0.02 \mu \mathrm{m}$ and $1.95 \pm 0.01 \mu \mathrm{m}$, respectively. They found the H5 Cangas de Onis chondrite as best match of their NIR spectrum, proposing 2007 PA8 as a second source of $\mathrm{H}$ chondrites, beside asteroid 6 Hebe.

The results based on our visible and near infrared spectral investigation confirm that 2007 PA8 has a young surface almost unweathered, but point toward a different meteorite analogy than that proposed by Nedelcu et al. (2014), i.e. with the $\mathrm{L}$ chondrites. Our data cover the full 0.4 to $2.4 \mu \mathrm{m}$ wavelength region, so we can better constrain the BI band spectral parameters and bands BII/BI area ratio compared to Nedelcu et al. (2014) work. We find a different BI center, situated at larger wavelength, in agreement with the mean BI band center reported in Dunn et al. (2010) for the $\mathrm{L}$ chondrites $(0.945-0.967 \mu \mathrm{m})$. If our and Nedelcu et al. (2014) BII center are similar, the shape and depth of this band is very different, our spectrum showing a much fainter absorption compared to them. The spectra were taken at similar phase angles (about $12^{\circ}$ ) and only one month apart, and any problem related to the instrumental and observing conditions or data reduction were found by us or reported by Nedelcu et al. (2014). Considering that 2007 PA8 is a large NEO with a long rotational period, and with an asymmetric shape having probably craters and boulders, as put in evidence by radar images (Brozovic et al., 2013), we interpret these spectral differences as due to heterogeneities in the surface composition of this slow rotator asteroid.

2007 PA8 is the Q-type asteroid with the largest semi major axis value in the known Q-type NEO population (Demeo et al. 2014). According to Bottke et al. (2002) NEO model, 2007 PA8 has a probability of 0.534 to have origin from the 
Jupiter family comet region, and a probability of 0.438 to come from the outer main asteroid belt (Nedelcu et al., 2014). Nedelcu et al. (2014) also perform some dynamical simulations showing that 2007 PA8 probably originated from a region bordering the 5:2 resonance in the outer belt (2.824 $\mathrm{AU})$, and that it rapidly enter in the NEO space. Indeed, the 5:2 resonance is the one that pumps orbital eccentricities on the shortest time scale, of the order of $10^{5}$ years (Yoshikawa, 1991; Ipatov 1992; Morbidelli \& Gladman 1998). Nedelcu et al. (2014) propose that 2007 PA8 was originated from the 5:2 resonance, then its eccentricity was rapidly pumped up, it crossed the main belt up to the Mars-crossing region, and it entered in the NEO space first as an Amor-class, then, five hundreds years ago, it became an Apollo-type. Close to the 5:2 resonance, two large families, Gefion and Koronis, dominated by S-complex asteroids have been identified.

The Gefion family was proposed as the probable source of L-type chondrites by Nesvorny et al. (2009) and Bottke et al. (2009) because of its proximity to the 5:2 resonance and because of its age, which is compatible with that of the catastrophic event of L-type meteorites parent body. Indeed, these chondrites represent about $47 \%$ of ordinary chondrite falls, and two third of them were heavily shocked near $470 \mathrm{Myr}$ ago, during the Ordovician period, probably as a consequence of the catastrophic disruption of their parent body at that time (Korochantseva et al. 2007, Haack et al. 1996, Heck, 2010). According to Nesvorny et al. (2009) studies, the Gefion family, located at 2.7-2.82 AU, has an estimated age of about $485 \mathrm{Myr}$, while the Koronis family is probably Gys old (Bottke et al. 2001). They also show that putative meteorites from the Gefion family may reach the NEO orbit in short time, and that the computed cosmic age exposure (CRE) of these simulated fragments should range from 5 to $100 \mathrm{Myr}$, with the peak of the dis- 
tribution around 30-40 Myr, that is closely matching the CRE of OC chondrites (Marti \& Graf, 1992). Faster Gefion meteorites reached the Earth between $50 \mathrm{Kyr}$ and 1-2 Myr after they had been placed in the 5:2 resonance, a time range compatible with the short CRE ages of fossil L chondrites observed in middle Ordovician sediments on Earth (Heck et al., 2004).

Considering both the dynamical works of Nesvorny et al. (2009) and Nedelcu et al. (2014), and the results of this paper indicating for 2007 PA8 a fresh surface, a Q-type classification, and a strong spectral similarity to L chondrites, we suggest that this PHA may be a member of the Gefion family recently ejected from the 5:2 resonance and a potential source of $\mathrm{L}$ chondrites.

\section{Acknowledgments}

The research leading to these results has received funding from the European Union Seventh Framework Program (FP7/2007-2013) under grant agreement No. 312430 (OPTICON). This paper is based on observations made with the Nordic Optical Telescope, operated by the Nordic Optical Telescope Scientific Association, and with the Italian Telescopio Nazionale Galileo (TNG) operated on the island of La Palma by the Centro Galileo Galilei of the INAF (Istituto Nazionale di Astrofisica), both located at the Observatorio del Roque de los Muchachos, La Palma, Spain, of the Instituto de Astrofisica de Canarias.” S.F. thanks Dr. V. Lorenzi, Dr. T. Augusteins and the TNG and NOT telescopes staff for their help in performing the observations. This project was supported by the French Planetology National Programme (INSU-PNP). D.P. acknowledge financial support from the NEOShield project, funded by the European Commission's Seventh Framework Programme (Contract No. FP7-SPACE-2011-282703). Part of this work 
was supported by the COST Action MP1104 "Polarization as a tool to study the Solar System and beyond". Taxonomic type results presented in this work were determined, in whole or in part, using a Bus-DeMeo Taxonomy Classification Web tool by Stephen M. Slivan, developed at MIT with the support of National Science Foundation Grant 0506716 and NASA Grant NAG5-12355. We thank R. Gil-Hutton and an anonymous referee for their comments who help us improving the paper.

\section{References}

Baffa, C., Comoretto, G., Gennari, S., Lisi, F., Oliva, E., Biliotti, V., Checcucci, A., Gavrioussev, V., Giani, E., Ghinassi, F., Hunt, L. K., Maiolino, R., Mannucci, F., Marcucci, G., Sozzi, M., Stefanini, P., Testi, L., 2001. NICS: The TNG Near Infrared Camera Spectrometer. Astron. Astroph. 378, 722-728

Belskaya, I.N., Fornasier, S., Krugly Y.N., 2009. Polarimetry and BVRI photometry of the potentially hazardous near-Earth Asteroid (23187) 2000 PN9. Icarus 201, 167-171

Belskaya, I. N., Shevchenko, V. G., 2000. Opposition effect of asteroids. Icarus 147, 94-105.

Bernardi, F., Micheli, M., Tholen, D. J., 2009. Absolute magnitude and slope parameter G calibration of asteroid 25143 Itokawa. Meteoritics \& Planet. Sci. 44, $1849-1852$

Binzel, R. P., Rivkin, A. S., Bus, S. J., Sunshine, J. M., Burbine, T. H., 2001. MUSES-C target asteroid (25143) 1998 SF36: A reddened ordinary chondrite. Meteoritics \& Planet. Sci. 36, 1167-1172

Bottke, W. F., Nesvorny, D., Vokrouhlicky, D., Morbidelli, A., 2009. The 
Gefion Family as the Probable Source of the L Chondrite Meteorites. 40th Lunar and Planetary Science Conference, id.1445

Bottke, W. F., Morbidelli, A., Jedicke, R., Petit, J. M., Levison, H. F., Michel, P., Metcalfe, T. S., 2002. Debiased Orbital and Absolute Magnitude Distribution of the Near-Earth Objects. Icarus, 156, 399-433

Bottke, W.F., Vokrouhlicky, D., Broz, M., Nesvorny, D., Morbidelli, A., 2001. Dynamical spreading of asteroid families by the Yarkovsky effect. Science 294, 1693-1696

Brozovic, M., Benner, L., Busch, M., Giorgini, J., Magri, C., 2013. Goldstone radar imaging and shape modeling of near-Earth Asteroid (214869) 2007 PA8. American Astronomical Society, DPS meeting 45, \#101.09

Cellino, A., Gil-Hutton, R., Tedesco, E. F., Di Martino, M., Brunini, A., 1999. Polarimetric observations of small asteroids: preliminary results. Icarus $138,129-$ 140.

Cellino, A., Gil-Hutton, R., Dell'Oro, A., Bendjoya, Ph., Canada-Assandri, M., Di Martino, M., 2012. A new calibration of the albedo - polarization relation for the asteroids. J. of Quantitative Spectroscopy and Radiative Transfer, 113, $2552-2560$

De Luise F., Perna D., Dotto E., Fornasier S., Belskaya, I. N., Boattini, A., Valsecchi, G. B., Milani, A., Rossi, A., Lazzarin, M., Paolicchi, P., Fulchignoni, M., 2007. Physical investigation of the potentially hazardous asteroid (144898) 2004 VD17. Icarus 191, 628-635

DeMeo, F.E., Binzel, R.P., Slivan, S.M., Bus, S.J., 2009. An Extension of the Bus Asteroid Taxonomy into the Near-Infrared. Icarus 202, 160-180.

DeMeo, F.E., Binzel, R.P., Lockharta, M., 2014. Mars encounters cause fresh 
surfaces on some near-Earth asteroids. Icarus 227, 112-122

Dunn, T. L., McCoy, T.J., Sunshine, J. M., McSween, H. Y., 2010. A coordinated spectral, mineralogical, and compositional study of ordinary chondrites. Icarus 208, 789-797

Dunn, T. L., Burbine, T. H., Bottke, W. F., Clark, J. P., 2013. Mineralogies and source regions of near-Earth asteroids. Icarus 222, 273-282

Fornasier, S., Dotto, E., Barucci, M. A., Barbieri, C., 2004. Water ice on the surface of the large TNO 2004 DW. Astron. Astroph. 422, L43-46.

Fornasier, S., Belskaya, I., Shkuratov, Yu. G., Pernechele, C., Barbieri, C., Giro, E., Navasardyan, H., 2006. Polarimetric survey of asteroids with the Asiago telescope. Astron. Astroph. 455, 371-377

Fornasier, S., Clark, B.E., Dotto, E., Migliorini, Ockert-Bell, M., Barucci, M. A., 2010. Spectroscopic survey of M-type asteroids. Icarus 210, 655-673

Fornasier, S., Clark, B.E., Dotto, E., 2011. Spectroscopic survey of X-type asteroids. Icarus 214, 131-146

Gaffey, M.J., Burbine, T.H., Piatek, J.L., Reed, K.L., Chaky, D.A., Bell, J.F., Brown, R.H., 1993. Mineralogical variations within the S-type asteroid class. Icarus 106, 573-602.

Geake, J. E., Dollfus, A., 1986. Planetary surface texture and albedo from parameter plots of optical polarization data. MNRAS 218, 75-91

Haack, H., Farinella, P., Scott, E.R.D., Keil, K., 1996. Meteoritic, asteroidal, and theoretical constraints on the $500 \mathrm{Ma}$ disruption of the L chondrite parent body. Icarus 119, 182-191.

Harris, A.W., 1998. A thermal model for near-Earth asteroids. Icarus 131, 291-301. 
Heck, P. R., Ushikubo, T., Schmitz, B., Kita, N. T., Spicuzza, M. J., Valley, J. W., 2010. A single asteroidal source for extraterrestrial Ordovician chromite grains from Sweden and China: High-precision oxygen three-isotope SIMS analysis. Geoch. Cosmoch. Acta 74, 497-509

Heck, P.R., Schmitz, B., Baur, H., Halliday, A.N., Wieler, R., 2004. Fast delivery of meteorites to Earth after a major asteroid collision. Nature 430, 323325 .

Hicks, M., Brewer, M., Somers, J., 2012. Broadband Photometry of (214869) 2007 PA8: A Slowly Rotating Potentially Hazardous Asteroid. The Astronomer's Telegram, \#4625

Korochantseva, E.V., Trieloff, M., Lorenz, C.A., Buykin, A.I., Ivanova, M.A., Schwarz, W.H., Hopp, J., Jessberger, E.K., 2007. L-chondrite asteroid breakup tied to Ordovician meteorite shower by multiple isochron 40Ar-39Ar dating. Meteorit. Planet. Sci. 42, 113-130.

Ipatov, S. I., 1992. Evolution of asteroidal orbits at the 5:2 resonance. Icarus, $95,100-114$

Lumme, K., Muinonen, K. 1993. A two-parameter system for linear polarization of some SolarSystem objects. Abstr. for the IAU Symposium 160, Asteroids, Comets, Meteors, p. 194

Jarosewich, E., 1990. Chemical analysis of meteorites: a compilation of stony and iron meteorites analysis. Meteoritics 15, 323-337.

Lupishko, D., Ed., Asteroid Polarimetric Database V8.0. EAR-A-3-RDRAPD-POLARIMETRY-V8.0. NASA Planetary Data System, 2014

Mainzer, A., Grav, T., Bauer, J. et al. 2011. NEOWISE observations of NearEarth objects: preliminary results. Astron. J. 743, 156 
Marti, K., Graf, T., 1992. Cosmic-ray exposure history of ordinary chondrites. Annu. Rev. Earth Planet. Sci. 20, 221-243.

Masiero, J.R., Mainzer, A.K., Grav, T., Bauer, J.M., Wright, E.L., McMillan, R. S., Tholen, D.J., Blain, A.W. 2012. A revised asteroid polarization-albedo relationship using WISE/NEOWISE data. Astroph. J. 749, 104, 6 pp.

Morbidelli, A., \& Gladman, B. 1998. Orbital and temporal distributions of meteorites originating in the asteroid belt. Meteor. Planet. Sci., 33, 999-1016

Nedelcu D. A., Birlan, M., Popescu, M., Badescu, O., Pricopi, D., 2014. Evidence for a second parent body of H-chondrite. Astron. Astroph. 567, L7, 5 $\mathrm{pp}$

Nesvorny, D., Vokrouhlicky, D. Morbidelli, A. Bottke, W. F., 2009. Asteroidal source of L chondrite meteorites. Icarus 200, 698-701

Oliva, T., 1997. Wedged double Wollaston, a device for single shot polarimetric measurements. Astron. Astroph. 123, 589-592

Perna, D., Barucci, M. A., Fulchignoni, M. 2013. The near-Earth objects and their potential threat to our planet. Astron. Astroph. Review, 21, id. 65

Pernechele, C., Fantinel, D., Giro, E., 2003. Device for optical linear polarization measurements with a single exposure. SPIE Proc., 4843, 156-163

Pieters, C. M., \& Hiroi, T., 2004. RELAB (Reflectance Experiment Laboratory): A NASA Multiuser Spectroscopy Facility. LPI, 35, 1720

Shakhovskoy, N. M. \& Efimov, Yu. S., 1972. Polarization observations of nonstable stars and extragalactic object. I: Equipment, method of observation and reduction. Izv. Krymskoi Astrofiz. Obs., 45, 90-110

Shoemaker, E. M., \& Helin, E. F., 1978. Earth-approaching asteroids as targets for exploration. NASA CP-2053, 245-256 
Tholen, D.J., Barucci, M.A., 1989. Asteroids taxonomy. In: Binzel, R.P., Gehrels, T., Matthews, M.S. (Eds.), Asteroids II. Univ. of Arizona Press, Tucson, pp. 298-315.

Thomas, C.A., Trilling, D. E., Emery, J. P., Mueller, M., Hora, J. L., Benner, L. A. M., Bhattacharya, B., Bottke, W. F., Chesley, S., Delbo, M., Fazio, G., Harris, A. W., Mainzer, A., Mommert, M., Morbidelli, A., Penprase, B., Smith, H. A., Spahr, T. B., Stansberry, J. A., 2011. ExploreNEOs. V. Average albedo by taxonomic complex in the near-Earth asteroid population. Astron. J. 142, id. 85, $12 \mathrm{pp}$

Yoshikawa, M. 1991. Motions of asteroids at the Kirkwood Gaps. II - On the 5:2, 7:3, and 2:1 resonances with Jupiter. Icarus, 92, 94-117

Zellner, B., Gradie, J. 1976. Minor planets and related objects. XX. Polarimetric evidence for the albedos and compositions of 94 asteroids. Astron. J. 81, 


\section{Tables}

Table 1: Orbital and physical characteristics of (214869) 2007 PA8. Orbital elements are taken from the JPL Small-Body Database. ${ }^{a}$ : Hicks et al. (2012), ${ }^{b}$ : Brozovic et al. (2013), ${ }^{c}$ : this paper.

\begin{tabular}{ll}
\hline perihelion distance (AU) & 0.955 \\
aphelion (AU) & 4.692 \\
semimajor axis (AU) & 2.823 \\
eccentricity & 0.662 \\
inclination (degrees) & 1.984 \\
synodical rotation period (hrs) & $95.1 \pm 3.4^{a}$ \\
& $101.6 \pm 2.0^{b}$ \\
absolute magnitude & $16.2 \pm 0.1$ \\
Tisserand param. & 2.947 \\
albedo & $0.29 \pm 0.08^{c}$ \\
diameter & $1.4 \pm 0.2^{c}$ \\
\hline
\end{tabular}


Table 2: Observing conditions of (214869) 2007 PA8 during the spectroscopic study in 2012 at the TNG telescope.

\begin{tabular}{|l|c|c|c|c|c|c|c|c|}
\hline Day & $\mathrm{UT}_{\text {start }}$ & Exp. (s) & Instrument & Airm & Solar An. (airm) & r $(\mathrm{AU})$ & $\delta(\mathrm{AU})$ & $\alpha\left(^{\circ}\right)$ \\
\hline 13 Oct. & $01: 44: 06$ & 180 & DOLORES-LRB & 1.23 & Land93-101(1.27) & 1.1552 & 0.1611 & 11.3 \\
13 Oct. & $01: 54: 02$ & 240 & DOLORES-LRR & 1.25 & Land93-101 (1.27) & 1.1551 & 0.1610 & 11.3 \\
13 Oct. & $02: 56: 02$ & 180 & DOLORES-LRB & 1.40 & Land93-101 (1.27) & 1.1546 & 0.1605 & 11.3 \\
13 Oct. & $03: 03: 59$ & 180 & DOLORES-LRR & 1.44 & Land93-101 (1.27) & 1.1545 & 0.1604 & 11.3 \\
12 Oct. & $23: 43: 31$ & 240 & NICS-Amici & 1.26 & Land93-101 (1.42) & 1.1555 & 0.1614 & 11.3 \\
\hline
\end{tabular}

\section{Figures}


Table 3: Observing conditions for the (214869) 2007 PA8 polarimetry in 2012. Data on the position angle of the scattering plane $(\phi)$, and on the phase angle $(\alpha)$ have been taken from the JPL ephemeris service (http://ssd.jpl.nasa.gov/cgi-bin/eph).

\begin{tabular}{|c|c|c|c|c|c|c|c|c|c|}
\hline DATE & $\mathrm{UT}_{\text {start }}$ & Band & $\begin{array}{l}\text { Exp. } \\
\text { (s) }\end{array}$ & $\begin{array}{c}\alpha \\
\left(^{o}\right)\end{array}$ & $\begin{array}{c}\phi \\
\left(^{o}\right)\end{array}$ & $\begin{array}{c}\mathrm{P} \\
(\%)\end{array}$ & $\left(^{o}\right)$ & $\begin{array}{l}\mathrm{P}_{r} \\
(\%)\end{array}$ & $\begin{array}{l}\theta_{r} \\
\left(^{o}\right)\end{array}$ \\
\hline 14 Oct. & $00: 26$ & B & 720 & 12.08 & 4.1 & $0.54 \pm 0.07$ & $179.3 \pm 4.1$ & $-0.53 \pm 0.07$ & $85.2 \pm 4.1$ \\
\hline 14 Oct. & $00: 40$ & V & 450 & 12.08 & 4.1 & $0.53 \pm 0.05$ & $189.6 \pm 4.4$ & $-0.53 \pm 0.05$ & $95.5 \pm 4.4$ \\
\hline 14 Oct. & $00: 48$ & $\mathrm{R}$ & 450 & 12.08 & 4.1 & $0.46 \pm 0.07$ & $191.5 \pm 5.3$ & $-0.44 \pm 0.07$ & $97.4 \pm 5.3$ \\
\hline 14 Oct. & $00: 58$ & I & 660 & 12.08 & 4.1 & $0.67 \pm 0.07$ & $191.2 \pm 4.0$ & $-0.65 \pm 0.07$ & $97.1 \pm 4.0$ \\
\hline 15 Oct. & $23: 28$ & B & 720 & 13.68 & 5.87 & $0.25 \pm 0.07$ & $180.7 \pm 4.8$ & $-0.25 \pm 0.07$ & $84.9 \pm 4.8$ \\
\hline 15 Oct. & $23: 38$ & V & 450 & 13.69 & 5.87 & $0.44 \pm 0.05$ & $192.5 \pm 3.4$ & $-0.43 \pm 0.05$ & $96.7 \pm 3.4$ \\
\hline 15 Oct. & $23: 47$ & $\mathrm{R}$ & 450 & 13.69 & 5.87 & $0.50 \pm 0.07$ & $182.0 \pm 4.0$ & $-0.49 \pm 0.07$ & $86.1 \pm 4.0$ \\
\hline 15 Oct. & $23: 56$ & $\mathrm{I}$ & 440 & 13.69 & 5.87 & $0.60 \pm 0.09$ & $199.7 \pm 5.8$ & $-0.53 \pm 0.09$ & $103.9 \pm 5.8$ \\
\hline 25 Oct. & 01:33 & B & 720 & 23.36 & -1.4 & $0.66 \pm 0.14$ & $91.9 \pm 6.1$ & $0.65 \pm 0.14$ & $3.3 \pm 6.1$ \\
\hline 25 Oct. & $01: 50$ & V & 450 & 23.36 & -1.4 & $0.41 \pm 0.10$ & $255.3 \pm 7.0$ & $0.35 \pm 0.10$ & $164.9 \pm 7.0$ \\
\hline 25 Oct. & 02:03 & $\mathrm{R}$ & 450 & 23.36 & -1.4 & $0.39 \pm 0.09$ & $252.3 \pm 9.7$ & $0.33 \pm 0.09$ & $163.7 \pm 9.7$ \\
\hline 25 Oct. & $02: 18$ & I & 660 & 23.36 & -1.4 & $0.30 \pm 0.07$ & $99.2 \pm 6.2$ & $0.28 \pm 0.07$ & $10.8 \pm 6.2$ \\
\hline 10 Nov. & $04: 38$ & B & 120 & 88.87 & 288.13 & $6.36 \pm 0.39$ & $197.3 \pm 2.0$ & $6.36 \pm 0.40$ & $179.2 \pm 2.0$ \\
\hline 14 Nov. & $05: 47$ & B & 360 & 99.22 & 285.15 & $7.00 \pm 0.27$ & $189.7 \pm 0.8$ & $6.95 \pm 0.27$ & $176.8 \pm 1.0$ \\
\hline 14 Nov. & $05: 54$ & V & 450 & 99.22 & 285.15 & $6.09 \pm 0.16$ & $191.9 \pm 1.2$ & $5.99 \pm 0.16$ & $175.0 \pm 1.2$ \\
\hline 14 Nov. & 06:03 & $\mathrm{R}$ & 450 & 99.22 & 285.15 & $5.62 \pm 0.25$ & $189.8 \pm 1.3$ & $5.42 \pm 0.25$ & $173.2 \pm 1.3$ \\
\hline 14 Nov. & $06: 13$ & I & 660 & 99.22 & 285.15 & $6.28 \pm 0.39$ & $187.7 \pm 1.8$ & $6.07 \pm 0.40$ & $172.6 \pm 1.8$ \\
\hline
\end{tabular}


Table 4: Parameters of the polarization phase curve and estimated values of the polarimetric albedo.

\begin{tabular}{|l|c|c|c|c|c|}
\hline Band & $\begin{array}{c}\mathrm{P}_{\min } \\
(\%)\end{array}$ & $\begin{array}{c}\text { slope (h) } \\
\left(\% /^{o}\right)\end{array}$ & $\begin{array}{c}\text { Inv. angle } \\
\left({ }^{o}\right)\end{array}$ & $\begin{array}{c}\mathrm{P}_{\max } \\
(\%)\end{array}$ & Albedo \\
\hline $\mathrm{B}$ & $<-0.53$ & $0.090 \pm 0.010$ & $17.7 \pm 1.1$ & $6.95 \pm 0.2$ & $0.25 \pm 0.06$ \\
$\mathrm{~V}$ & $<-0.52$ & $0.078 \pm 0.010$ & $19.0 \pm 1.1$ & $5.99 \pm 0.16$ & $0.29 \pm 0.08$ \\
$\mathrm{R}$ & $<-0.44$ & $0.073 \pm 0.010$ & $19.3 \pm 1.1$ & $5.42 \pm 0.25$ & $0.31 \pm 0.09$ \\
$\mathrm{I}$ & $<-0.65$ & $0.083 \pm 0.008$ & $20.2 \pm 1.1$ & $6.07 \pm 0.40$ & $0.27 \pm 0.07$ \\
\hline
\end{tabular}




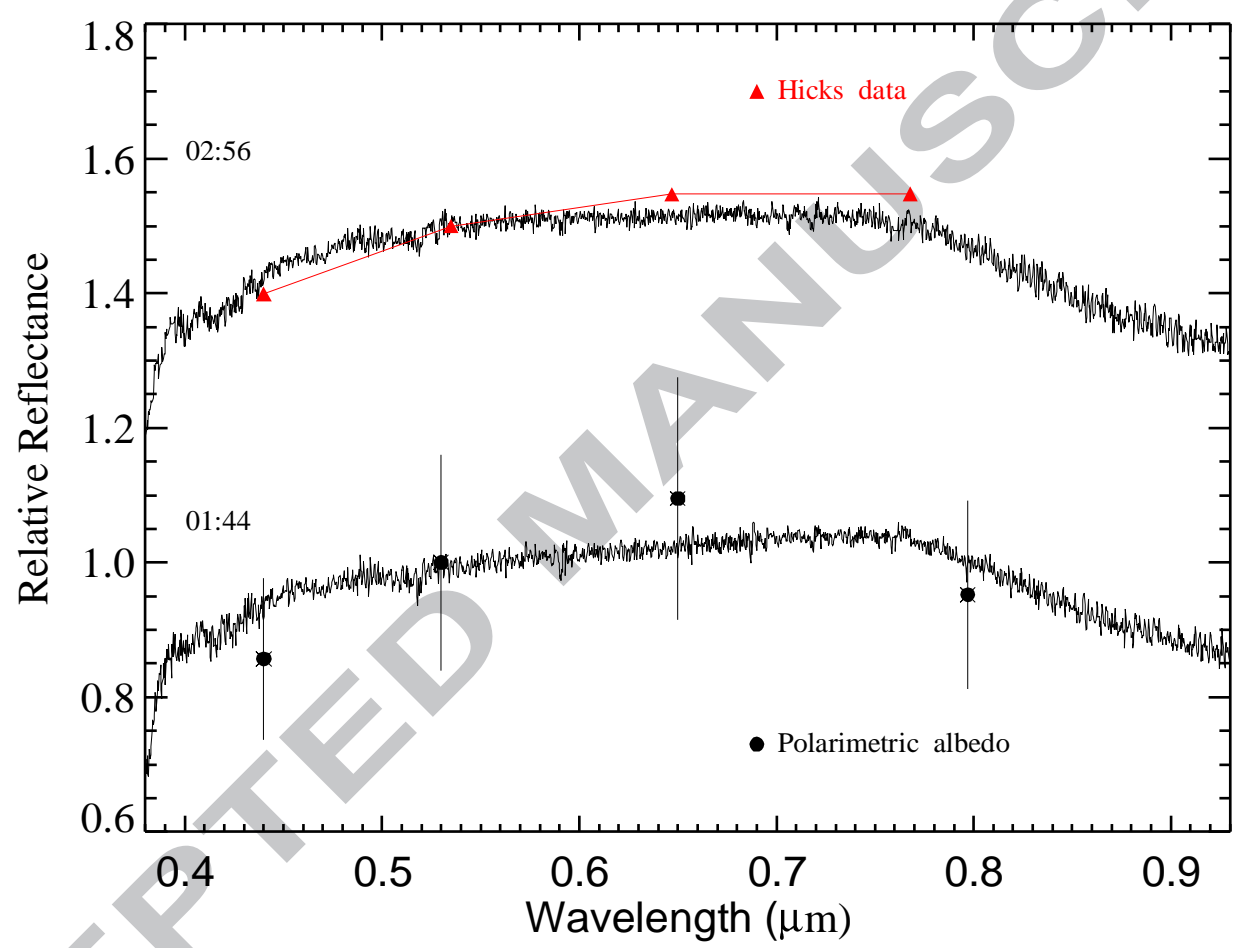

Figure 1: Visible spectra of 2007 PA8. The albedo from B, V, R, and I polarimetry, normalized in the $\mathrm{V}$ band, is superposed to the spectrum acquired at 01:44. The spectrophotometry from the Hicks et al. (2012) B, V, R, and I data is superposed to the spectrum acquired at 02:56. 


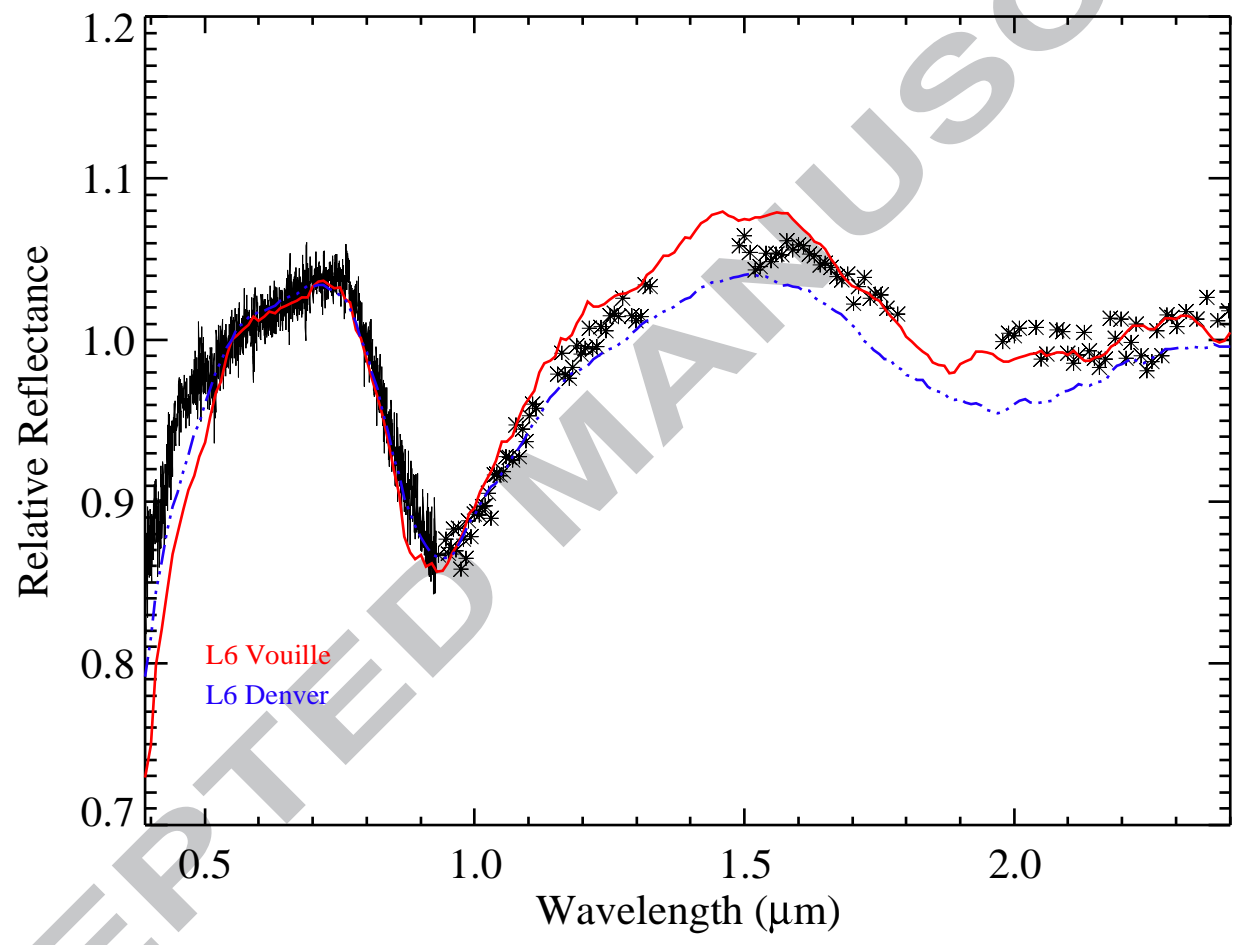

Figure 2: Visible and near infrared spectrum of 2007 PA8 with overplotted the two L6 chondrites that best fit the data (Vouille meteorite: continuous line; Denver meteorite: dashed-dot line). 


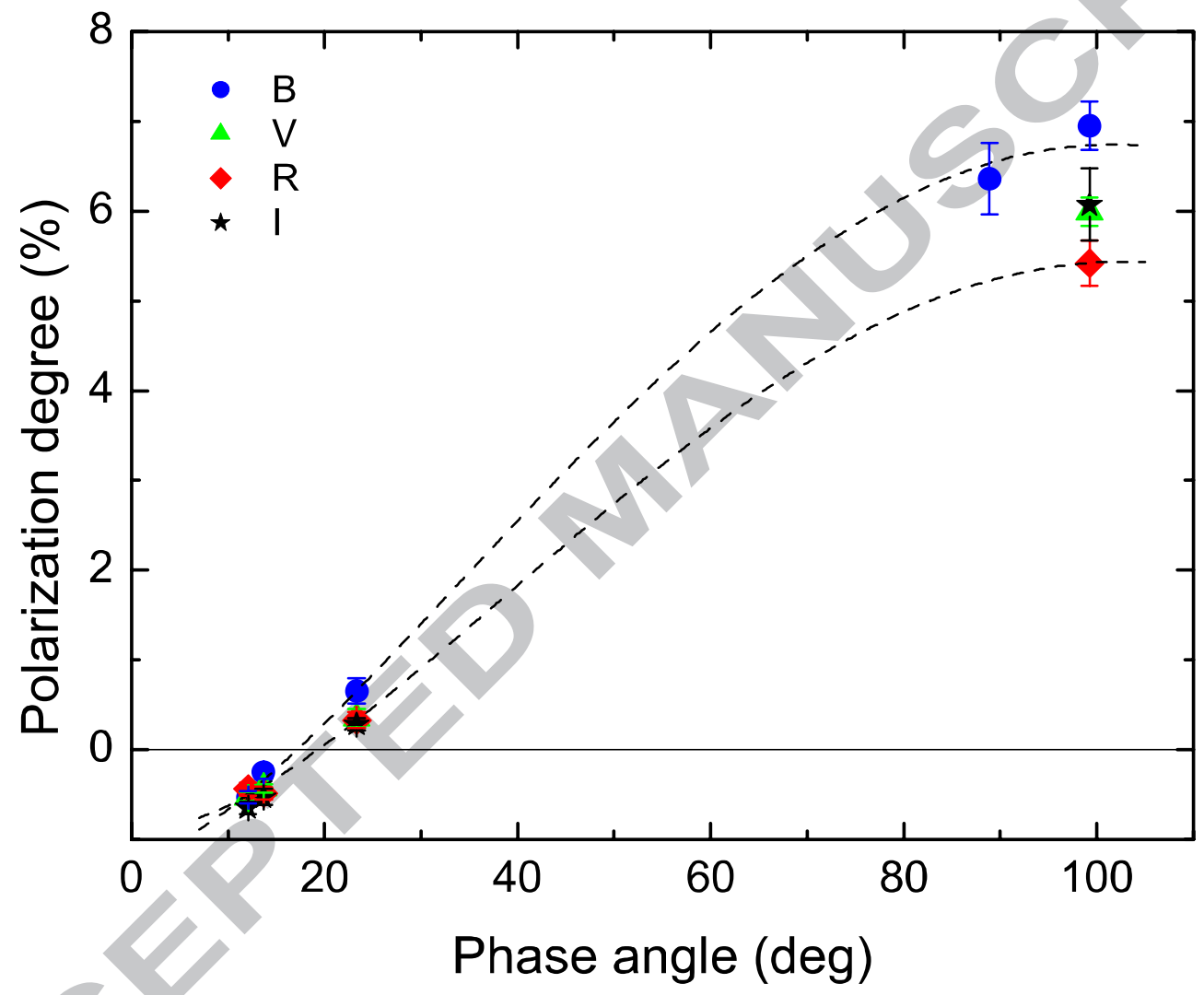

Figure 3: Polarization phase angle dependence measured in the BVRI band for the NEA (214869) 2007 PA8. The fit with the Lumme and Muinonen function (Lumme \& Muinonen, 1993) is shown for the $\mathrm{B}$ and $\mathrm{R}$ bands (dashed lines). 


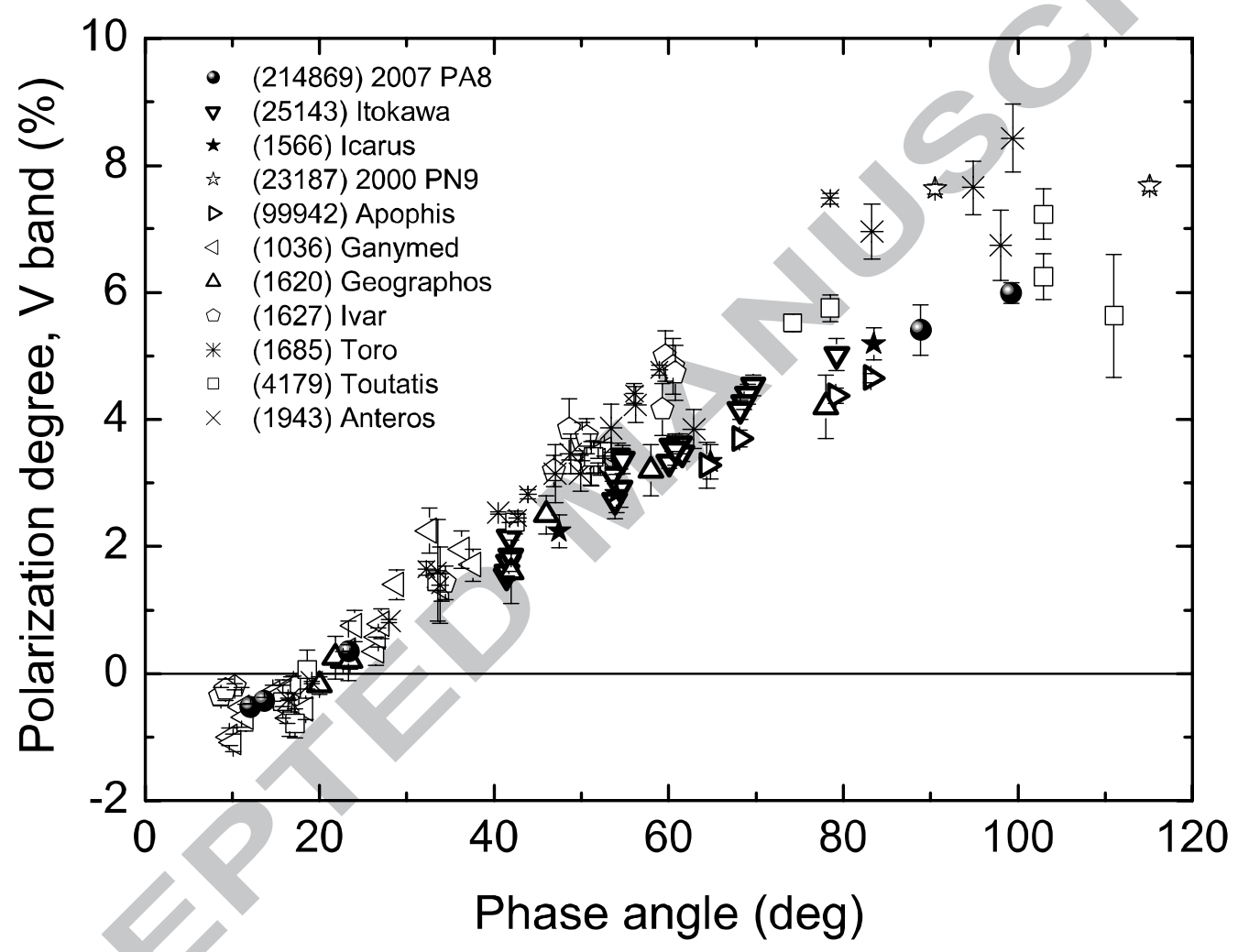

Figure 4: Polarization phase dependence for moderate albedo asteroids. The data were taken from the Asteroid Polarimetric Database compiled by Lupishko (2014). Our observations of 2007 PA8 are shown by the black circles. 


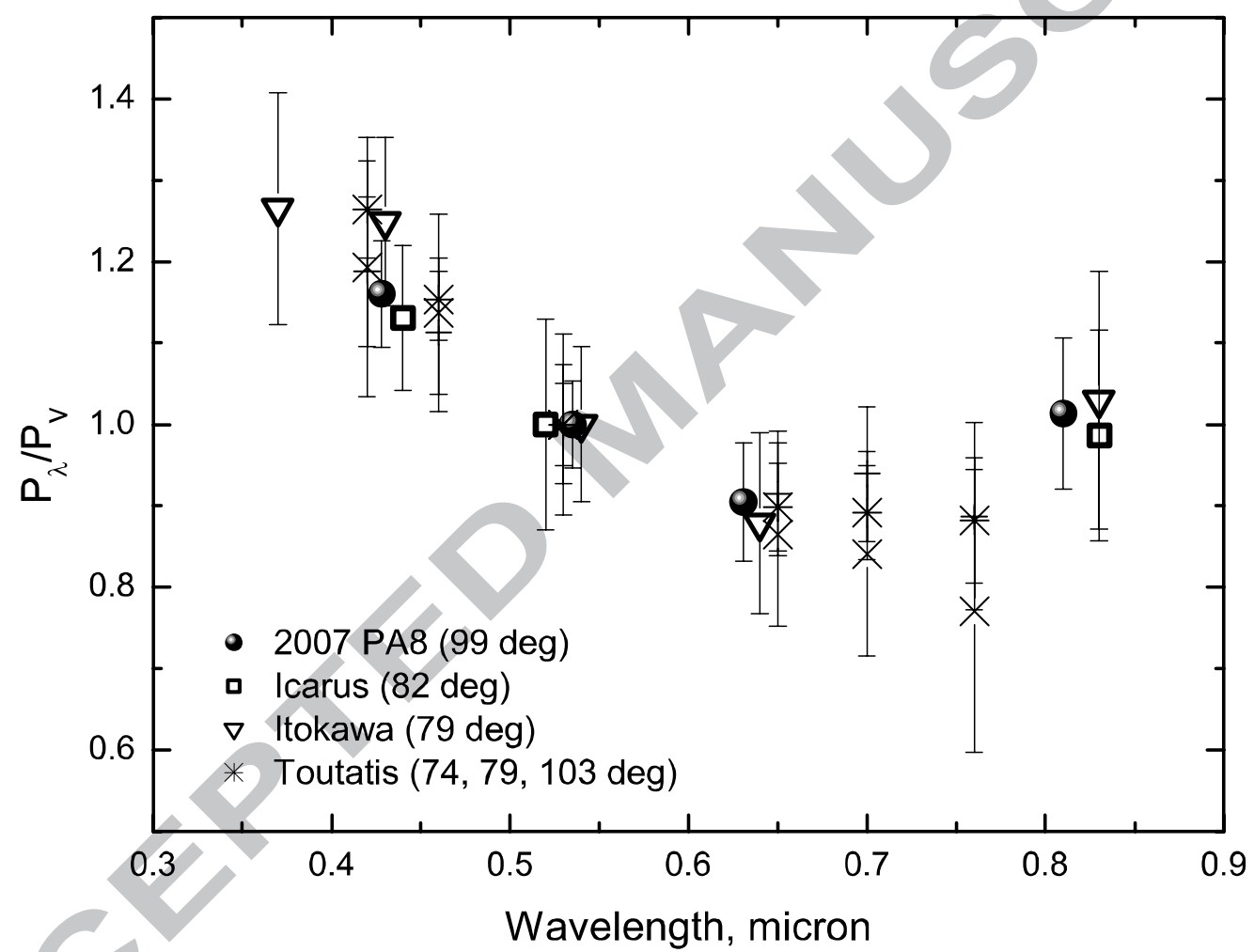

Figure 5: Wavelength dependence of polarization for NEAs. The phase angle is also shown. 


\section{Highlights}

- We present the results from polarimetry and spectroscopy of the PHA 214869 (2007 PA8)

- The spectra reveal that 2007 PA8 is a Q-type

- Spectral parameters and meteorites comparison indicate a L chondrites analogy

- We derive a geometric albedo of $0.29 \pm 0.08$ and a diameter of $1.4 \pm 0.2 \mathrm{~km}$

- 2007 PA8 may be a member of the Gefion family recently ejected from the 5:2 resonance 